\title{
S-allylmercaptocysteine promotes MAPK inhibitor-induced apoptosis by activating the TGF- $\beta$ signaling pathway in cancer cells
}

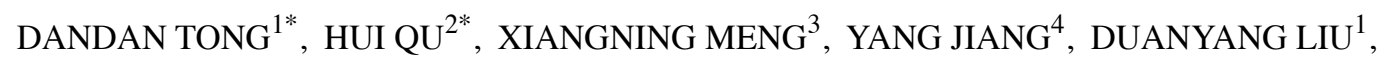 \\ SHENGQIAN YE $^{1}$, HE CHEN ${ }^{1}$, YAN JIN ${ }^{3,5}$, SONGBIN FU ${ }^{3,5}$ and JINGSHU GENG ${ }^{3,4}$
}

\author{
${ }^{1}$ Department of Pathology, Harbin Medical University; ${ }^{2}$ Department of Pediatrics, The Second Affiliated Hospital, \\ Harbin Medical University; ${ }^{3}$ Department of Medical Genetics, Harbin Medical University; ${ }^{4}$ Department of Pathology, \\ The Third Affiliated Hospital, Harbin Medical University; ${ }^{5}$ Key Laboratory of Medical Genetics (Harbin Medical \\ University), Heilongjiang Higher Education Institutions, Harbin, Heilongjiang 150081, P.R. China
}

Received February 24, 2014; Accepted May 23, 2014

DOI: 10.3892/or.2014.3295

\begin{abstract}
S-allylmercaptocysteine (SAMC), one of the water-soluble organosulfur garlic derivatives, can induce the apoptosis of many types of cancer cells through the MAPK signaling pathway. The TGF- $\beta$ signaling pathway also plays a pivotal role in the process of oncogenesis, and has a certain crosstalk with the MAPK pathway. In the present study, hepatocellular carcinoma cell line HepG2 with an intact TGF- $\beta$ signal and colon cancer cell line SW620 with an imperfect TGF- $\beta$ signal were selected to ascertain whether SAMC induces the apoptosis of cancer cells by TGF- $\beta$ signaling. In both cell lines treated with MAPK inhibitors and SAMC, an increased apoptosis rate was observed by electron microscopy, TUNEL and flow cytometric assays. Immunohistochemistry and western blot assays showed that SAMC induced the apoptosis of cancer cells by activating TGF- $\beta 1, T \beta R I I, p-s m a d 2 / 3$, smad4 and smad7 signals, and promoting Bim expression while decreasing Bcl-2 expression and finally activating the mitochondrial apoptosis pathway proteins caspase-3 and caspase-9 in the HepG2 cell line. In contrast, in the SW620 cell line, the apoptosis induced by SAMC only affected TGF- $\beta 1$ and smad7 signals, and promoted the expression of Bax and Bad and finally activated the mitochondrial apoptosis
\end{abstract}

Correspondence to: Professor Songbin Fu, Medical Genetics Laboratory, Harbin Medical University, Harbin, Heilongjiang 150081, P.R. China

E-mail: fusb@ems.hrbmu.edu.cn

Professor Jingshu Geng, Department of Pathology, Third Affiliated Hospital, Harbin Medical University, Harbin, Heilongjiang 150081, P.R. China

E-mail: jingshugeng@me.com

${ }^{*}$ Contributed equally

Key words: S-allylmercaptocysteine, apoptosis, TGF- $\beta$ pathway, smad, Bim pathway protein caspase-9. When we compare the apoptosis rate in both cell lines, a significantly lower apoptosis rate was noted in the SW620 cell line than the rate noted in the HepG2 cell line. In summary, SAMC induces the apoptosis of cancer cells by activating the TGF- $\beta$ signaling pathway, after MAPK signaling is inhibited.

\section{Introduction}

Allicin (diallyl thiosulfinate) is the major component in garlic (Allium sativum) extract and it has multiple antineoplastic and other pharmacological effects. The antitumor activities of allicin include the inhibition of cell proliferation and the induction of apoptosis in multiple types of malignancies, including colon, breast and lung cancer (1-5). Allicin has both lipid- and water-soluble components. Lipid-soluble compounds of allicin include diallyl sulfide (DAS), diallyl disulfide (DADS) and diallyl trisulfide (DATS), whereas watersoluble components of allicin consist of S-allylcysteine (SAC), S-allylmercaptocysteine (SAMC) and other components.

The functions of SAMC and other organic sulfur compounds derived from garlic have been compared. Among the 10 garlic-derived compounds tested, SAMC, DADS and S-trityl-L-cysteine were shown to markedly inhibit cell proliferation, cause G2-M arrest and induce apoptosis (6). Although DAS, DADS, DATS, SAC and SAMC are organic sulfur compounds with similar functions, SAMC showed the most significant effect in promoting apoptosis, including apoptosis in the colon cancer cell lines, HT-29 and SW620 (4).

Studies have shown that allicin induces apoptosis mainly through a mitogen-activated protein kinase (MAPK) signaling cascade (4,6-9). The MAPK signaling cascade possesses a bi-directional mode of action for regulating tumor cell growth, proliferation and apoptosis according to environmental conditions. This cascade includes signal-related kinase (ERK), c-jun-N-terminal kinases (JNK) and the p38 MAPK pathways. Shirin et al (4) and Xiao et al (10) demonstrated that, by activating JNK1 and caspase-3, SAMC inhibited proliferation and induced the apoptosis of colon cancer cells $(4,10)$. In addition, 
Chen et al demonstrated that the three main MAPKs (ERK, JNK and p38) are activated after treatment of human hepatoma cells with DATS (11).

The ability to inhibit apoptosis via the MAPK signaling cascade is shared by SP600125, SB203580 and PD98059, which inhibit JNK, P38K and ERK, respectively (12-14). It has been reported that the synergistic actions of these inhibitors with allicin result in higher rates of apoptosis than when these inhibitors act alone. Examples of this synergy include SB203580 enhancement of DADS-induced apoptosis in hepatocellular carcinoma (HepG2 cells) (13) and more efficient induction of apoptosis when the inhibitors (SB203580 and PD98059) act cooperatively with allicin and SAMC in the treatment of colon cancer (cell line SW480) versus the independent actions of these agents (4).

The transforming growth factor $\beta$ (TGF- $\beta$ ) signaling pathway plays important roles in tumorigenesis (15-21) and has extensive interactions with the MAPK signaling pathways (4,6-9). In addition to the TGF- $\beta$ pathway, the cytokine TGF- $\beta 1$ activates the MAPK pathways. As a consequence, activated ERK, JNK and P38 can phosphorylate Smad2/3 and Smad4 to facilitate their translocation to the nucleus $(22,23)$. Thus, it is possible that the TGF- $\beta$ signaling pathway participates in the allicin-induced apoptosis of tumor cells. However, this has yet to be established.

The TGF- $\beta$ signaling pathway in hepatocellular carcinoma HepG2 cells has been extensively studied. However, the roles of the TGF- $\beta$ pathway in the inhibition of cell proliferation or apoptosis induction have not been extensively studied (24). The TGF- $\beta$ pathway may induce apoptosis when the MAPK pathway is inhibited. Caja et al reported that the TGF- $\beta$ pathway did not induce apoptosis in HepG2 cells until the MEK/ERK pathway was repressed (25). The TGF- $\beta$ pathway was found to be inactivated in the SW620 colon cancer cell line due to the lack of Smad4 protein (24), while reactivation occurred after transfecting the cells with Smad4. Based on these observations, we aimed to ascertain whether the TGF- $\beta$ signaling pathway is activated by SAMC to induce apoptosis in HepG2 and SW620 cells. We then examined the specific role of the TGF- $\beta$ pathway in apoptosis induction by further suppressing the MAPK pathway.

\section{Materials and methods}

Experimental reagents. SAMC was generously provided by Wakunaga Pharmaceutical Company, Hiroshima, Japan. ERK inhibitor PD98059 (cat. no. 513000), JNK inhibitor 600125 (cat. no. 420119) and P38 inhibitor SB203580 (cat. no. 559389) were from Calbiochem. The Annexin V-FITC Apoptotic Kit and the In Situ Cell Death Detection Kit were purchased from BD and Roche, respectively. The following primary antibodies were used for immunoblotting and were purchased from Santa Cruz: anti-TGF- $\beta 1$, anti-T $\beta$ R1, anti-T $\beta$ R2, anti-phosphorylated Smad2/3, anti-Smad4, anti-Bim, anti-Bcl-2, anti-Bax, antiBad, anti-caspase-3 and anti-caspase-9. Secondary anti-rabbit and anti-goat antibodies were from Zhongshan Golden Bridge Biotechnology. The following parallel experiments were conducted using both the colon cancer cell line SW620 and the liver cancer cell line HepG2. The SAMC solution was newly prepared before each use.
Cell culture. The colon cancer cell line SW620 and the liver cancer cell line HepG2 obtained from the American Type Culture Collection (ATCC, Manassas, VA, USA) were maintained in Dulbecco's modified Eagle's medium (DMEM) supplemented with $10 \%$ fetal bovine serum (FBS; Gibco) and incubated at $37^{\circ} \mathrm{C}$. We maintained a total of four sets of cultured cells: the control set received only DMEM, whereas the other sets received SAMC (group S), MAPK inhibitors (group I) and SAMC combined with the MAPK inhibitors (group I+S). The MAPK inhibitors consisted of three inhibitors, each at the concentration of $10 \mu \mathrm{g} / \mathrm{ml}$, which blocked ERK (PD98059), JNK (600125) and P38 (SB203580). All groups of cells were treated for $8 \mathrm{~h}$. Specifically, the cells in group I+S were pretreated with a combination of MAPK inhibitors for $30 \mathrm{~min}$ before receiving $800 \mu \mathrm{mol} / 1 \mathrm{SAMC}$ for an additional $8 \mathrm{~h}$.

Apoptosis was detected using electron microscopy (EM). After the cells had grown to $85 \%$ confluency in 100-ml Petri dishes, they were treated with the MAPK inhibitors and/or SAMC for $8 \mathrm{~h}$ as described above. Next, the cells were trypsinized, centrifuged and washed twice with PBS. The resulting cell pellets were fixed with $2.5 \%$ glutaraldehyde for $24 \mathrm{~h}$ at $4^{\circ} \mathrm{C}$, sectioned at $50 \mathrm{~nm}$ thickness, stained on copper screens and observed by EM. Ten microscopic fields $(x 3,000)$ were randomly chosen for each sample, and the apoptotic cells were counted.

Flow cytometry. Cells in an exponential growth stage were seeded in 6-well plates at a density of $5 \times 10^{6}$ cells/well and incubated at $37^{\circ} \mathrm{C}$ until the cells reached $85-90 \%$ confluency. Following an 8-h treatment with the MAPK inhibitors and/or SAMC, the cells were trypsinized, washed twice with cold PBS and then washed once with the binding buffer. Annexin V and PI staining were performed according to the manufacturer's instructions. The cells were analyzed by flow cytometry within $2 \mathrm{~h}$ to detect apoptotic cells.

Immunocytochemistry. Sterilized coverslips were placed in 24-well plates, and $2 \times 10^{5}$ cells in an exponential growth stage were seeded in each well onto the coverslips. At 85-90\% confluency, the cells received the same treatments (i.e., MAPK inhibitors and/or SAMC) for $8 \mathrm{~h}$ as previously described. The cells were then fixed with $4 \%$ newly prepared paraformaldehyde solution for $10 \mathrm{~min}$ at room temperature, followed with two 10-min washes with PBS, and incubated with $0.1 \%$ Triton X-100 on ice for 2 min before additional PBS washes.

To detect cellular apoptosis, the samples were incubated with $100 \mu \mathrm{l}$ of TUNEL reaction solution and Tdt enzyme at $37^{\circ} \mathrm{C}$ for $1 \mathrm{~h}$ in a dark humid environment, while the negative control received only $100 \mu \mathrm{l}$ of the TUNEL label solution. TUNEL-positive apoptotic cells were detected by fluorescence microscopy. We randomly chose 10 microscopic fields (x100) and counted the apoptotic cells. The rate of cellular apoptosis was expressed as a percentage of the total cells.

To detect protein expression and phosphorylation, the samples were incubated separately with a 1:50 dilution of primary antibodies, including those of anti-TGF- $\beta 1$, anti-T $\beta$ R1, anti-T $\beta$ R2, anti-phosphorylated Smad2/3, anti-Smad4, antiBcl-2, anti-Bax, anti-Bad, anti-caspase-3, anti-caspase-9 or with a $1: 100$ dilution of anti-Bim at $37^{\circ} \mathrm{C}$ for $1 \mathrm{~h}$, followed by 
PBS washes and incubation with the secondary antibody at room temperature for $20 \mathrm{~min}$. The samples were then washed with PBS, stained with DAB and hematoxylin dye according to the standard protocols. After gradient dehydration, the samples were mounted with Neutral gum.

The immunohistochemistry results were assessed using the following standards. Brown-yellow granules in the cytoplasm indicated positive expression of TGF- $\beta 1, T \beta R 1, T \beta R 2$ and Smad4; brown particles in the nucleus and the cytoplasm indicated positive expression of P-Smad2/3, Bim and Bax. For each sample, 10 microscopic fields were chosen (x100), and both positive and negative cells were counted. Staining intensity was divided into four grades according to the proportion of positive cells to the total cell numbers: the number of positive cells $<10 \%(-), 10-25 \%(+), 26-50 \%(++),>50 \%(+++)$.

Western blot analysis. Cultured cells were lysed with the T-PER tissue protein extraction reagent in the presence of protease inhibitors (Pierce, Rockford, IL, USA). Equal amounts of cellular proteins were loaded into each well and resolved using 10\% SDS-PAGE gels. Nitrocellulose membrane blotting was then performed under standard conditions. For immunoblotting, we used primary antibodies against TGF- $\beta 1$, T $\beta R 1$, T $\beta R 2$, phosphorylated Smad2/3, Smad4, Bcl-2, Bax, Bad, caspase-3, caspase- 9 and Bim. The primary antibody solution was diluted at room temperature and incubated with the membrane on a swing bed for $1 \mathrm{~h}$. The membrane was then washed 3 times for 10 min with TBS-T before adding a 1:2,000 dilution of the secondary antibody, which was diluted in the blocking solution at room temperature. The membrane was then incubated on a swing bed for $1 \mathrm{~h}$ before three $10-\mathrm{min}$ washes with TBS-T.

Statistical analysis. All data are reported as the mean \pm SD. A one-way analysis of variance was used to test the differences among three or more groups (SAS 9.2 package). For all statistically significant differences observed after performing one-way analysis of variance, the appropriate multiple comparison tests were used to perform a post-hoc analysis. Statistical significance was set at $\mathrm{P}<0.05$.

\section{Results}

Detection of SAMC-induced cell apoptosis by EM. Apoptosis is the process of programmed cell death that is characterized by cell shrinkage, nuclear condensation, nuclear fragmentation and formation of apoptotic bodies (26). First, we observed the morphological changes in tumor cells by EM to determine the impact of allicin, MAPK inhibitors or the combined treatment of SAMC and MAPK inhibitors on the tumor cells. The characteristic markers of the control HepG2 and SW620 cells were as follows: surface rich in microvilli; abundant cytoplasm, mitochondria and endoplasmic reticulum, low electron density, large and irregular nuclei, distinct nuclear membranes and large nucleoli near the nuclear membranes (Fig. 1A and B). Cells from group $\mathrm{S}$ were characterized as follows: decreased or absent membrane microvilli, smaller cell size, concentrated cytoplasm with increased electron density, expanded and vacuolized endoplasmic reticulum, nuclear chromatin that was gathered at the nuclear membranes and visible apoptotic bodies, which contained pyknotic nuclear remains and organelles that were encapsulated by the cell membranes. Compared with the control group, we detected apoptotic cells and fewer proliferating cells in the inhibitor group. Evident mitochondrial vacuolation and an increased number of apoptotic cells were observed in group I+S compared with the other groups. There were no significant differences among all the groups $(\mathrm{P}>0.05)$.

TUNEL assay reveals the highest number of apoptotic cells in group $I+S$. TUNEL assay results showed that the number of apoptotic cells was increased $(\mathrm{P}<0.05)$ in the HepG2 cells from group $\mathrm{S}$ compared with those from the control group. Group I+S had the largest number of apoptotic cells when compared with the control group, and the difference was statistically significant $(\mathrm{P}<0.0001)$. There was no difference in the rate of apoptosis between group I and the control group $(\mathrm{P}>0.05)$. The number of apoptotic cells was increased in the SW620 cells from group S compared with those from the control group $(\mathrm{P}>0.05)$. Group $\mathrm{I}+\mathrm{S}$ had an increased number of apoptotic cells compared with this number in the control group, and the difference was statistically significant $(\mathrm{P}<0.001)$. There was no significant difference in the cellular apoptotic rate when comparing group I with the control group $(\mathrm{P}>0.05)$ (Fig. 1A-C).

Flow cytometry results showed that cells in groups $S, I$ and $I+S$ underwent early-stage apoptosis; group $I+S$ exhibited the most significant induction of apoptosis. In HepG2 cells, the late-stage apoptotic rates were 2.52,1.66 and 3.12\% in group S, group I and group I+S, respectively, compared with the control group rate of $0.17 \%$; the results indicated that there was a significant increase in the number of apoptotic cells following treatment $(\mathrm{P}<0.05)$ (Fig. 2). The early-stage apoptotic rates of groups S, I, I+S and the control group were 18.03, 17.88, 27.74 and $2.87 \%$, respectively; the number of apoptotic cells also increased after treatment and the results indicated that there was a significant increase in the number of apoptotic cells after treatment $(\mathrm{P}<0.05)$. In the SW620 cells, the late-stage apoptotic rates were $1.80,1.33,1.35$ and $0.06 \%$ in groups $\mathrm{S}, \mathrm{I}, \mathrm{I}+\mathrm{S}$ and the control group, respectively; the number of apoptotic cells increased after treatment and this increase was statistically significant $(\mathrm{P}<0.05)$. The early-stage apoptotic rates were $17.80,15.79$ and $26.84 \%$ in groups S, I and I+S, respectively, when compared with $0.37 \%$ of the control group; the number of apoptotic cells increased and the difference was statistically significant $(\mathrm{P}<0.05)$. The number of apoptotic cells increased in group $\mathrm{I}+\mathrm{S}$ and was statistically significant when compared with that of group $\mathrm{S}(\mathrm{P}<0.05)$.

Immunohistochemical results. In HepG2 cells from the group I+S, TGF- $\beta 1$, phosphorylated $\mathrm{Smad} 2 / 3$ and Smad4 and caspase-3 expression levels were 1+ (Fig. 3A); whereas their expression levels were negative or not evident and the differences in the protein expression levels were not significant when comparing group $\mathrm{I}+\mathrm{S}$ with the other three groups $(\mathrm{P}>0.05)$. T $\beta \mathrm{R} 2$, Bim, and caspase-9 expression levels were $2+$ in group $\mathrm{I}+\mathrm{S}$ while the other groups were negative and $1+$; the differences in the expression levels of these proteins were statistically significant when comparing group $\mathrm{I}+\mathrm{S}$ with 

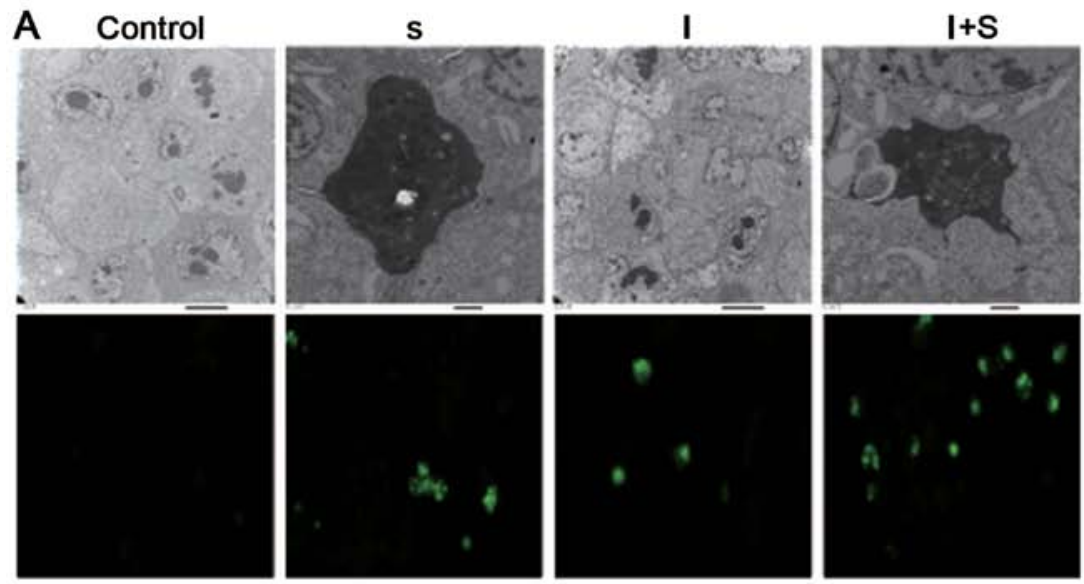

HepG2

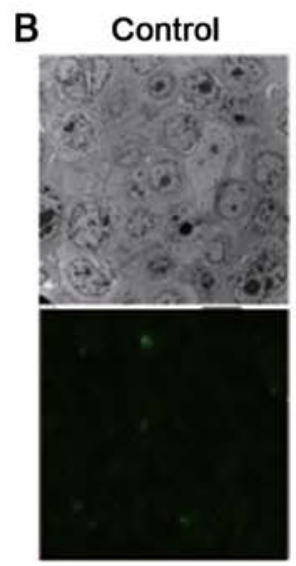

$\mathbf{S}$

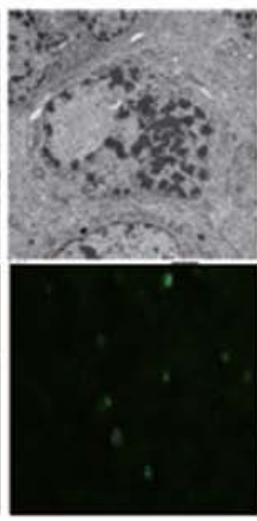

I

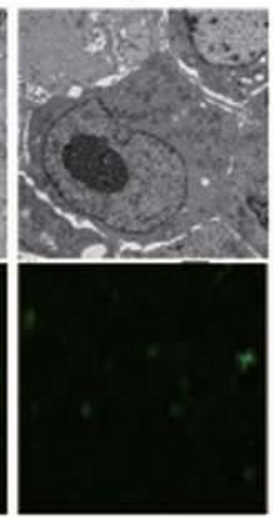

$1+S$

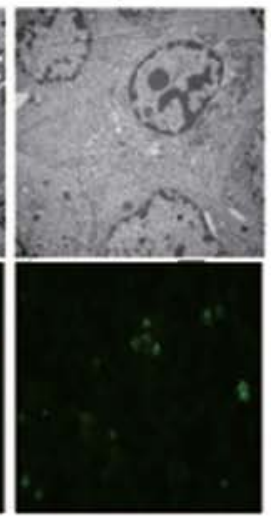

SW620

C

HepG2

SW620

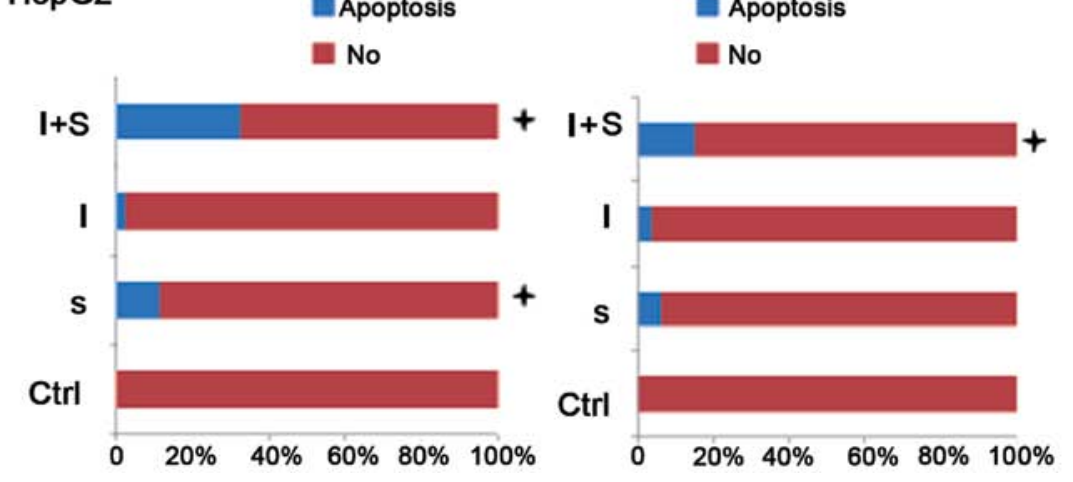

Figure 1. Apoptosis analysis by electron microscopy and TUNEL staining of the four treatment groups: control, S, I, I+S. (A) Results for apoptosis by electron microscopy; magnification, x3,000. Groups S, I and I+S in the HepG2 cells showed evident apoptosis when the HepG2 and SW620 cells were treated with SAMC and the MAPK inhibitors. (B and C) Statistical results for apoptosis by TUNEL analysis indicated that there were significantly higher apoptotic rates in the HepG2 cells from groups S and I+S than in the other groups (magnification, $\mathrm{x} 400$ ). In the SW620 cells, group I+S showed a significantly higher apoptotic rate when compared with the other groups.

the control group $(\mathrm{P}<0.05)$. In the SW620 cells from group $\mathrm{S}$, $\mathrm{T} \beta \mathrm{R} 2$ expression increased to $1+$ as compared with the control group but the difference did not achieve statistical significance $(\mathrm{P}>0.05)$ (Fig. 3B). TGF- $\beta 1, \mathrm{~T} \beta \mathrm{R} 2$, Smad4 and caspase-9 protein expression was increased in group I+S compared with the control group, but the differences in protein levels did not achieve statistical significance $(\mathrm{P}>0.05)$.
Western blotting results. In the HepG2 cells, Smad4 expression increased and Smad7 expression decreased in group S compared with the cells from the control group (Fig. 4A and B). The expression of TGF- $\beta 1$ and Smad 4 decreased while the Bcl-2 protein levels were elevated in group I as compared with the control group. TGF- $\beta 1, T \beta R I I$, phosphorylated Smad2/3, Smad4, Smad7, Bim, caspase-3 and caspase-9 
A

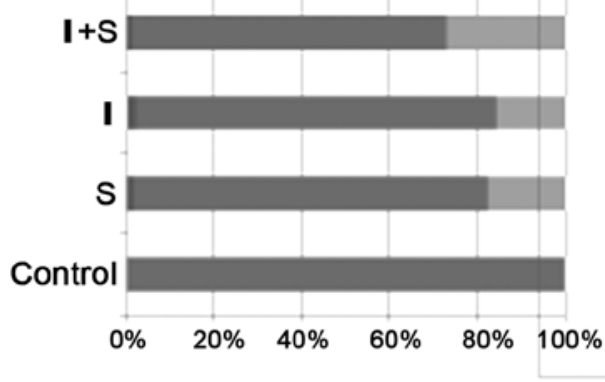

B

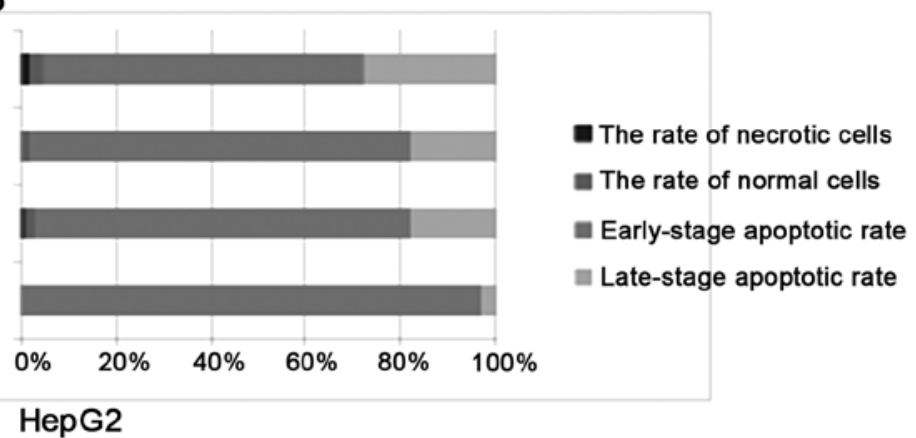

Figure 2. Analysis of apoptosis by flow cytometry. The number of cells in the early apoptotic stages were increased with either SAMC or MAPK inhibitor treatment. The percentage of apoptotic cells in groups S, I and I+S exhibited significant differences when compared with the control group. Similar results were obtained for the SW620 and HepG2 cells.

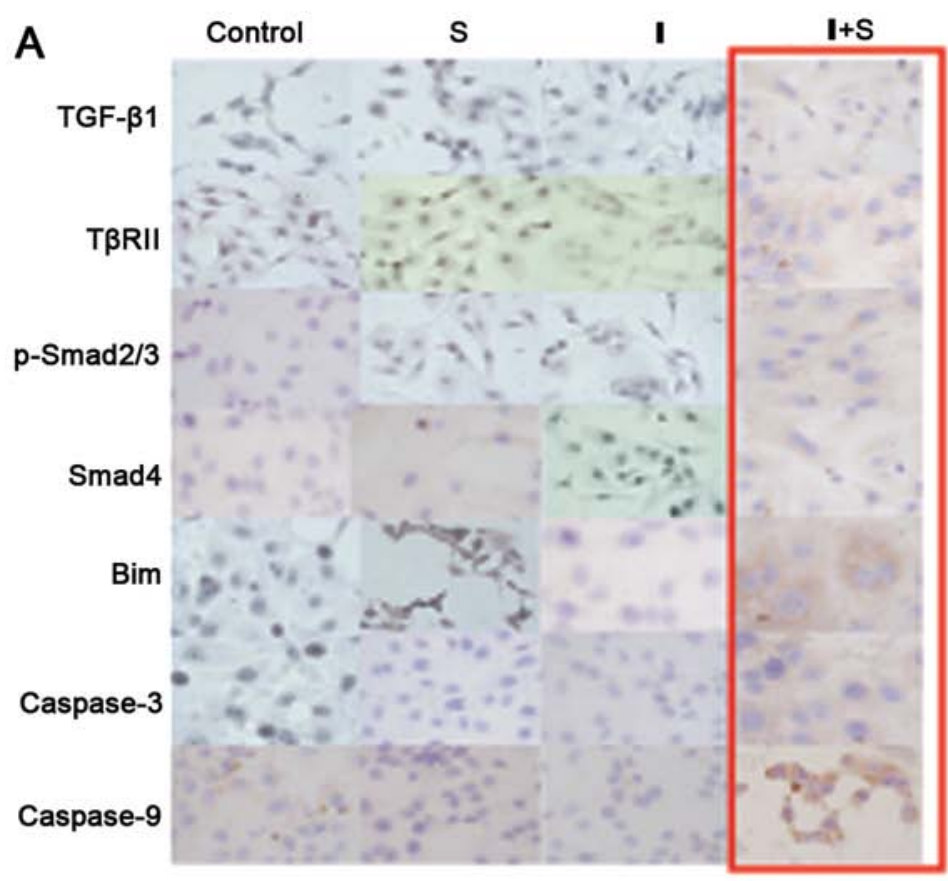

HepG2

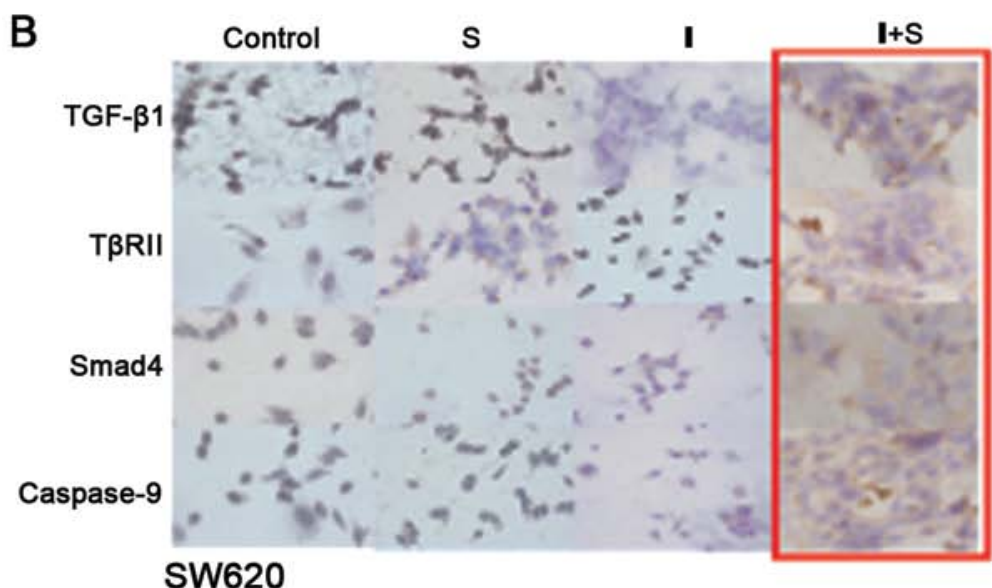

Figure 3. Immunocytochemical analysis (magnification, $\mathrm{x} 400$ ). (A) In the HepG2 cells from group I+S , the expression levels of TGF- $\beta 1$, p-Smad2/3, Smad4 and caspase-3 were 1+, the expression levels of T $\beta$ RII, Bim and caspase-9 were 2+, whereas the expression of the same proteins were negative or 1+ in cells from the other groups. (B) In the SW620 cells, the expression level of T $\beta$ RII was 1+ in group S and was high when compared with the level in the control group; the expression levels of TGF- $\beta 1$, T $\beta$ RII, Smad4 and caspase- 9 were increased in group I+S compared with the level in the control group. 
A

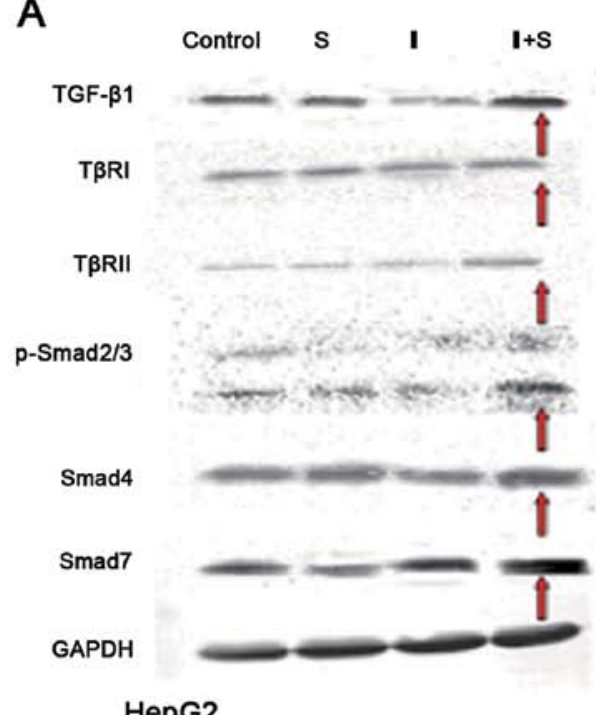

HepG2

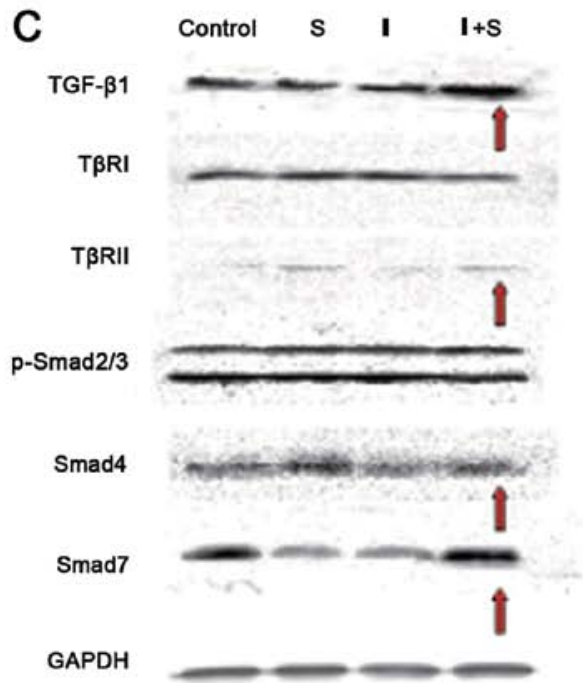

SW620
B

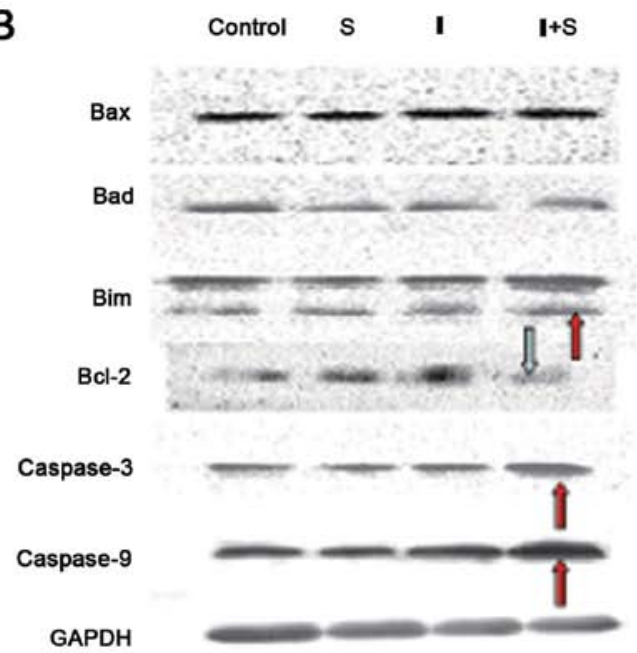

HepG2

D

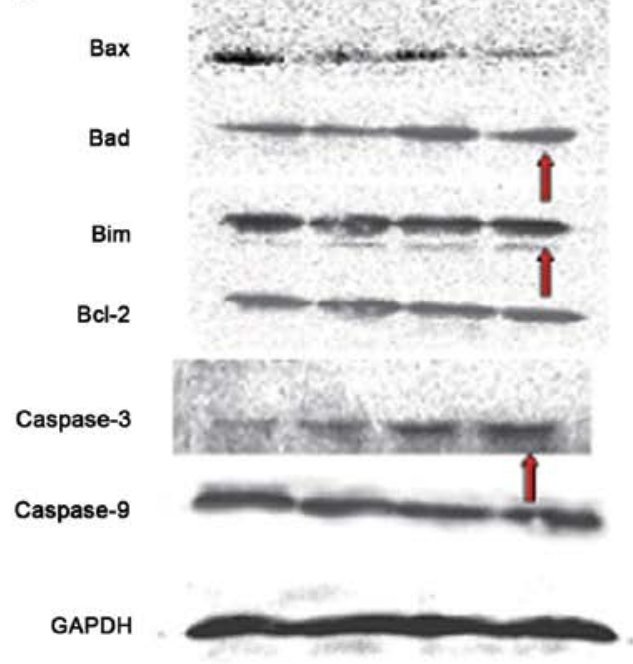

SW620

Figure 4. Western blot analysis. (A and B) In the HepG2 cells, increased Smad4 expression and decreased Smad7 expression were detected in group S compared with the control cells. The expression levels of TGF- $\beta 1$, T $\beta$ RII, p-Smad2/3, Smad4, Smad7, Bim, caspase-3 and caspase-9 were increased while Bcl-2 expression was decreased in group I+S compared with group S. (C and D) In the SW620 cells, TRRII, Smad4 and Bax expression levels were increased while Smad7 expression was decreased in group S compared with the control group. The expression levels of TGF- $\beta 1$, Smad7, Bax, Bad and caspase-9 were increased in group I+S as compared with those in group S.

expression increased while Bcl-2 decreased in the HepG2 cells from group I+S compared with those from group $\mathrm{S}$.

In the SW620 cells from group S, the expression of T $\beta R I I$ increased and Smad4, Bax and Smad7 expression decreased as compared with the cells from the control group. Smad7 expression was decreased in group I compared with that in the control group. TGF- $\beta 1$, Smad7, Bax, Bad and caspase-9 expression increased in group $\mathrm{I}+\mathrm{S}$ compared with group $\mathrm{S}$ (Fig. 4C and D).

\section{Discussion}

SAMC-induced apoptosis of HepG-2 and SW620 cells. Mechanisms of the suppression of cancer by allicin include inhibition of cell proliferation, induction of cell cycle arrest, induction of cancer cell differentiation and apoptosis $(2,4,10$, 27-29), direct killing of cancer cells and other functions. The inhibition of cell proliferation and the induction of apoptosis are the most significant functions. When HepG2 and SW620 cancer cells were treated with water-soluble allicin SAMC, their apoptotic rates were increased compared with the untreated cells. These results are consistent with previous studies performed in SW480, SNU-1, HEL and OCIM-1 cells $(2,6,10)$, which indicate that SAMC induces apoptosis in multiple cancer cells.

The MAPK signaling cascade is an important intracellular signaling pathway that regulates cell growth, and its inhibitors can induce cancer cell apoptosis (12-14). In the present study, 
we treated HepG2 and SW620 cells with three inhibitors of the MAPK pathway (JNK, ERK and P38MAPK) and each inhibitor induced obvious signs of apoptosis. Therefore, the activation of the intrinsic MAPK signaling pathway contributed to the protection of the cancer cells and the inhibition of apoptosis.

We subsequently combined the inhibitors of the MAPK pathway with SAMC to treat the cancer cells. This combined treatment resulted in significantly higher apoptotic rates than the treatment with SAMC or the inhibitors alone $(\mathrm{P}<0.05)$. In this case, higher apoptotic rates were observed in the HepG2 cells than in the SW620 cells. These results indicated that the induction of apoptosis by the MAPK inhibitors was synergistically enhanced with SAMC. Previous studies have shown that the collaborative action of inhibitors and allicin induced higher apoptotic rates than that of each single factor $(10,13)$. Our results also demonstrated that the rate of SAMC-induced apoptosis was the highest when the MAPK pathway was suppressed. Allicin induces apoptosis via the MAPK pathway. Therefore, there remains the question of whether allicin induces apoptosis via other pathways when the MAPK pathway is blocked by inhibitors. To elucidate this issue, we examined the TGF- $\beta$ signaling pathway, which is closely related to the MAPK pathway.

SAMC activation of the TGF- $\beta$ signaling pathway following suppression of the MAPK pathway. Intracellular signaling transduction composes a complex network and there is communication between the TGF- $\beta$ and MAPK signaling pathways $(30,31)$. Perturbances of the TGF- $\beta$ signaling pathway could lead to the occurrence and development of various types of tumors (15-21). For instance, the TGF- $\beta$ pathway was found to be inactivated in 20 out of 38 cancer cell lines (24) and there was also low or no expression of several proteins such as T $\beta$ RII and Smad4. Furthermore, the activity of the TGF- $\beta$ pathway could be partially recovered by the transfection of downregulated genes. We observed that SAMC activates the TGF- $\beta$ signaling pathway and induces apoptosis in HepG2 cells with intact TGF- $\beta$ pathways (32) and also in SW620 cells with perturbed TGF- $\beta$ pathways (24).

SAMC activation of TGF- $\beta 1$ with MAPK pathway suppression. Our results indicated that SAMC alone was not able to activate TGF- $\beta 1$ in HepG2 and SW620 cells. This suggests that SAMC may induce apoptosis via other signaling pathways, such as the MAPK pathway (4,6-9). We observed that the expression of TGF- $\beta 1$ was decreased after the treatment of MAPK inhibitors in HepG2 cells. These results are consistent with those of previous studies where PD98059 and SB203580 repressed the expression of TGF- $\beta 1$ in mouse macrophages (33). However, TGF- $\beta 1$ could be activated by SAMC when the MAPK pathway is inhibited with concurrent upregulation of proteins in the TGF- $\beta$ pathway. Therefore, given the complex interactions among the various intracellular signaling pathways, it is possible for SAMC to promote the expression of TGF- $\beta 1$ through pathways other than MAPK in the presence of inhibitors.

The expression of many proteins in the TGF- $\beta$ pathway was increased in the HepG 2 cells, whereas only TGF- $\beta 1$, T $\beta$ RII and Smad4 proteins were upregulated in the SW620 cells. We believe this upregulation occurred as the TGF- $\beta$ pathway is intact in HepG2 cells, which enables SAMC to activate the TGF- $\beta$ pathway when the MAPK pathway is completely inhibited. Conversely, the TGF- $\beta$ pathway was blocked in the SW620 cells resulting in insufficient pathway activation, despite using comparable treatments.

Re-expression or upregulation of inactivated Smad4 by SAMC in cancer cells. Smad4 is a tumor-suppressor gene whose function is to initiate TGF- $\beta$-induced tumorigenesis and metastasis. Miyaki et al discovered that the inactivation of the Smad4 gene results in tumor development, invasion and metastasis, and the stable transfection of Smad4 into SW620 cells could inhibit cancer cell migration and metastasis while reducing the occurrence of tumors (34). Our results showed that the expression of Smad4 was increased following SAMC treatment and Smad4 expression decreased when HepG2 cells were treated with the MAPK inhibitors, whereas combined treatments increased Smad4 expression levels, when compared with the SAMC treatment alone. The expression of Smad4 was correlated with the TGF- $\beta 1$ levels, suggesting that TGF- $\beta 1$ initiates changes in cells with active TGF- $\beta$ signaling pathways that result in the expression of downstream Smad4 proteins (35-37). In addition, we discovered that SAMC induces the re-expression of inactivated Smad4 genes in SW620 cells, despite the non-obvious changes in TGF- $\beta 1$ levels. The expression of Smad4 may be achieved by both TGF- $\beta$-dependent and TGF- $\beta$-independent pathways. These results suggest that regulation of Smad 4 could occur by the TGF- $\beta$ signaling pathway, the MAPK and other pathways (38-40). Therefore, we propose that SAMC induces expression of Smad4 through other mechanisms as well as the regulation by SAMC of the TGF- $\beta$ signaling pathway.

Dual functions of Smad7. Smad7 is considered to be an inhibitory protein in the TGF- $\beta$ signaling pathway. TGF- $\beta 1$ may suppress apoptosis by interacting with active T $\beta$ RI to further activate Smad7, which subsequently inhibits the phosphorylation of $\mathrm{p}-\mathrm{Smad}$ and thus suppresses the TGF- $\beta$ signaling pathway $(41,42)$. However, our results showed that, when the MAPK signaling pathway was inhibited by SAMC, there was an increase in the expression of endogenous TGF- $\beta 1$, Smad7 and other proteins that promote apoptosis. Similarly, Landström et al discovered that treating human prostate cancer cells with TGF- $\beta 1$ or the ectopic overexpression of Smad7 induced apoptosis (43), and Mazars et al reported that Smad7 induced apoptosis in HepG2 cells (44). However, we speculated that the induction of apoptosis by Smad7 is dependent on the cell type. Regardless, the potential for Smad7 to both inhibit and induce apoptosis is a novel insight into the characteristics of Smad7.

SAMC activation of the TGF- $\beta$-mitochondrial pathway for the induction of apoptosis following MAPK pathway inhibition. Activation of the TGF- $\beta$ pathway promotes apoptosis initiation and progression by supporting pro-apoptotic factors while the anti-apoptotic factors (such as Bcl-2 family proteins) are destroyed. The induction of apoptosis varies with each cell type. For example, in the breast cancer cell lines JygMC(A) and JygMC(B), TGF- $\beta$ upregulated the Bim pathway through Foxcl (45) while TGF- $\beta$ downregulated the Bcl-2 pathway 
through Smad3 in the lung cancer cell line A549 (46); and TGF- $\beta 1$ upregulated Bim and Bax in osteoclast cells (47). During the induction of apoptosis, changes in the anti-apoptotic Bcl-2 family proteins and pro-apoptotic proteins such as Bax, Bak and Bim play an important role. We examined the Bcl-2 family and caspase proteins, which are located downstream of the TGF- $\beta$ pathway. TGF- $\beta 1$ activated the expression of p-Smad2/3, Smad4 and Bim proteins in HepG2 cells, when the MAPK pathway was inhibited by SAMC treatment, similarly to previous reports on WEHI231B lymphocytes $(19,48)$. The expression of $\mathrm{Bcl}-2$ protein decreased as the expression of Bim increased and Bim interacted with Bcl-2 to induce the caspase cascade via a change in the conformation of the complex (49). When the expression of $\mathrm{Bcl}-2$ was reduced, the ratio of $\mathrm{Bcl}-2 /$ Bax decreased leading to apoptosis (50). Our results also demonstrated that the cells entered apoptosis when the expression levels of caspase-3 and caspase-9 were elevated, as evident by cell shrinkage, chromatin condensation, karyopyknosis, nuclear fragmentation and an increased rate of apoptosis.

Although the TGF- $\beta$ pathway in HepG 2 cells is intact, cell proliferation and induction of apoptosis are not believed to be inhibited (24). Our study demonstrated that SAMC induced apoptosis by activating the TGF- $\beta$ signaling pathway when the MAPK pathway was suppressed.

Wen et al also noted that SB203580, when combined with DADS, enhanced the DADS-induced apoptosis of HepG2 cells (13). In the present study, we discovered that SAMC further induced cell apoptosis by activating the TGF- $\beta$ signaling cascade and subsequently increased the expression levels of apoptotic protein after the ERK, JNK and p38K pathways were inhibited.

In the SW620 cells, when the MAPK signaling cascade was suppressed, SAMC mainly stimulated the expression of Bax and Bad proteins, but not Bim. These results indicated that SAMC was able to promote apoptosis by partially activating the TGF- $\beta$ cascade, thereby increasing the expression levels of Bax and caspase-9 in SW620 cells, whose TGF- $\beta$ pathway was otherwise inactivated. These results are similar to a study performed by Lee et al, who reported that SAMC induced the apoptosis of SUN-1 cells by activating Bax (2).

The TGF- $\beta$ pathway is inactivated in many tumor cells and tissues. Although the TGF- $\beta$ signaling pathway can function normally in most hepatic carcinoma cell lines, these cells do not undergo apoptosis via this signaling pathway (24). Notably, after the MAPK pathway was suppressed, SAMC further induced cell apoptosis by reactivating the TGF- $\beta$ pathway. The combined effects of the MAPK inhibitors and SAMC included higher induction rates of tumor cell apoptosis when compared with either treatment alone. These results help to elucidate the underlying mechanism and the possible signal networks that are implicated in allicin-induced tumor cell apoptosis. MAPK inhibitors have been tested in clinical trials, and the clinical use of SAMC has been approved in China. Our results support the translational and clinical applications of combining MAPK inhibitor and SAMC in cancer therapy.

\section{Acknowledgements}

This study was supported by the National Natural Science Foundation of China (no. 81372785), the Natural Science
Foundation of Heilongjiang (nos. D200921 and QC2009C29), the Opening Project of the Key Laboratory of Medical Genetics (Harbin Medical University), Heilongjiang Higher Education Institutions. We thank Wakunaga Pharmaceutical, Hiroshima, Japan, for the gift of the garlic-derived compound SAMC to Professor Yong-Chuan Wong, University of Hong Kong.

\section{References}

1. Hu H, Zhang XP, Wang YL, et al: Identification of a novel function of Id-1 in mediating the anticancer responses of SAMC, a water-soluble garlic derivative, in human bladder cancer cells. Mol Med Rep 4: 9-16, 2011.

2. Lee Y: Induction of apoptosis by S-allylmercapto-L-cysteine, a biotransformed garlic derivative, on a human gastric cancer cell line. Int J Mol Med 21: 765-770, 2008.

3. Pinto JT, Lapsia S, Shah A, Santiago H and Kim G: Antiproliferative effects of garlic-derived and other allium related compounds. Adv Exp Med Biol 492: 83-106, 2001.

4. Shirin H, Pinto JT, Kawabata Y, et al: Antiproliferative effects of S-allylmercaptocysteine on colon cancer cells when tested alone or in combination with sulindac sulfide. Cancer Res 61: 725-731, 2001.

5. Wu X, Kassie F and Mersch-Sundermann V: Induction of apoptosis in tumor cells by naturally occurring sulfur-containing compounds. Mutat Res 589: 81-102, 2005.

6. Xiao D, Pinto JT, Gundersen GG and Weinstein IB: Effects of a series of organosulfur compounds on mitotic arrest and induction of apoptosis in colon cancer cells. Mol Cancer Ther 4: 1388-1398, 2005.

7. Malki A, El-Saadani M and Sultan AS: Garlic constituent diallyl trisulfide induced apoptosis in MCF7 human breast cancer cells. Cancer Biol Ther 8: 2175-2185, 2009.

8. Wu XJ, Hu Y, Lamy E and Mersch-Sundermann V: Apoptosis induction in human lung adenocarcinoma cells by oil-soluble allyl sulfides: triggers, pathways, and modulators. Environ Mol Mutagen 50: 266-275, 2009.

9. Zhang W, Ha M, Gong Y, Xu Y, Dong N and Yuan Y: Allicin induces apoptosis in gastric cancer cells through activation of both extrinsic and intrinsic pathways. Oncol Rep 24: 1585-1592, 2010.

10. Xiao D, Pinto JT, Soh JW, et al: Induction of apoptosis by the garlic-derived compound S-allylmercaptocysteine (SAMC) is associated with microtubule depolymerization and c-Jun NH(2)-terminal kinase 1 activation. Cancer Res 63: 6825-6837, 2003.

11. Chen C, Pung D, Leong V, et al: Induction of detoxifying enzymes by garlic organosulfur compounds through transcription factor Nrf2: effect of chemical structure and stress signals. Free Radic Biol Med 37: 1578-1590, 2004.

12. Bennett BL, Sasaki DT, Murray BW, et al: SP600125, an anthrapyrazolone inhibitor of Jun N-terminal kinase. Proc Natl Acad Sci USA 98: 13681-13686, 2001.

13. Wen J, Zhang Y, Chen X, Shen L, Li GC and Xu M: Enhancement of diallyl disulfide-induced apoptosis by inhibitors of MAPKs in human HepG2 hepatoma cells. Biochem Pharmacol 68: 323-331, 2004.

14. Xia HH, He H, De Wang J, et al: Induction of apoptosis and cell cycle arrest by a specific c-Jun NH2-terminal kinase (JNK) inhibitor, SP-600125, in gastrointestinal cancers. Cancer Lett 241: 268-274, 2006.

15. Herzer K, Grosse-Wilde A, Krammer PH, Galle PR and Kanzler S: Transforming growth factor-beta-mediated tumor necrosis factor-related apoptosis-inducing ligand expression and apoptosis in hepatoma cells requires functional cooperation between Smad proteins and activator protein-1. Mol Cancer Res 6: 1169-1177, 2008.

16. Korchynskyi O, Landström M, Stoika R, et al: Expression of Smad proteins in human colorectal cancer. Int $\mathrm{J}$ Cancer 82: 197-202, 1999.

17. Perlman R, Schiemann WP, Brooks MW, Lodish HF and Weinberg RA: TGF-beta-induced apoptosis is mediated by the adapter protein Daxx that facilitates JNK activation. Nat Cell Biol 3: 708-714, 2001.

18. Teraoka H, Sawada T, Yamashita Y, et al: TGF-beta1 promotes liver metastasis of pancreatic cancer by modulating the capacity of cellular invasion. Int J Oncol 19: 709-715, 2001. 
19. Wildey GM, Patil S and Howe PH: Smad3 potentiates transforming growth factor beta (TGFbeta)-induced apoptosis and expression of the BH3-only protein Bim in WEHI 231 B lymphocytes. J Biol Chem 278: 18069-18077, 2003.

20. Yang YA, Zhang GM, Feigenbaum L and Zhang YE: Smad3 reduces susceptibility to hepatocarcinoma by sensitizing hepatocytes to apoptosis through downregulation of Bcl-2. Cancer Cell 9: 445-457, 2006.

21. Yu J, Zhang L, Chen A, et al: Identification of the gene transcription and apoptosis mediated by TGF-beta-Smad2/3-Smad4 signaling. J Cell Physiol 215: 422-433, 2008.

22. Chapnick DA, Warner L, Bernet J, Rao T and Liu X: Partners in crime: the TGF $\beta$ and MAPK pathways in cancer progression. Cell Biosci 1: 42, 2011.

23. Javelaud D and Mauviel A: Crosstalk mechanisms between the mitogen-activated protein kinase pathways and Smad signaling downstream of TGF-beta: implications for carcinogenesis. Oncogene 24: 5742-5750, 2005.

24. Ijichi H, Ikenoue T, Kato N, et al: Systematic analysis of the TGF-beta-Smad signaling pathway in gastrointestinal cancer cells. Biochem Biophys Res Commun 289: 350-357, 2001.

25. Caja L, Sancho P, Bertran E, Iglesias-Serret D, Gil J and Fabregat I: Overactivation of the MEK/ERK pathway in liver tumor cells confers resistance to TGF-\{beta\}-induced cell death through impairing up-regulation of the NADPH oxidase NOX4. Cancer Res 69: 7595-7602, 2009.

26. Harada K, Kawaguchi S, Supriatno, Kawashima Y, Yoshida H and Sato M: S-1, an oral fluoropyrimidine anti-cancer agent, enhanced radiosensitivity in a human oral cancer cell line in vivo and in vitro: involvement possibility of inhibition of survival signal, Akt/PKB. Cancer Lett 226: 161-168, 2005.

27. Chu Q, Lee DT, Tsao SW, Wang X and Wong YC: S-allylcysteine, a water-soluble garlic derivative, suppresses the growth of a human androgen-independent prostate cancer xenograft, CWR22R, under in vivo conditions. BJU Int 99: 925-932, 2007.

28. Howard EW, Lee DT, Chiu YT, Chua CW, Wang X and Wong YC: Evidence of a novel docetaxel sensitizer, garlicderived S-allylmercaptocysteine, as a treatment option for hormone refractory prostate cancer. Int J Cancer 122: 1941-1948, 2008.

29. Liang D, Qin Y, Zhao W, et al: S-allylmercaptocysteine effectively inhibits the proliferation of colorectal cancer cells under in vitro and in vivo conditions. Cancer Lett 310: 69-76, 2011.

30. Guo $X$ and Wang XF: Signaling cross-talk between TGF-beta/BMP and other pathways. Cell Res 19: 71-88, 2009.

31. Xu J, Lamouille S and Derynck R: TGF-beta-induced epithelial to mesenchymal transition. Cell Res 19: 156-172, 2009.

32. Tong JL, Nie F, Ran ZH, et al: Epigallocatechin gallate induces apoptosis in human hepatocellular carcinoma HepG2 cells via TGF/Smad signaling pathway. Zhonghua Zhong Liu Za Zhi 31: 646-650, 2009 (In Chinese).

33. Wang L, Hu GY, Shen H, Peng ZZ, Ning WB and Tao LJ: Fluorofenidone inhibits TGF-beta1 induced CTGF via MAPK pathways in mouse mesangial cells. Pharmazie 64: 680-684, 2009.
34. Miyaki M, Iijima T, Konishi M, et al: Higher frequency of Smad4 gene mutation in human colorectal cancer with distant metastasis. Oncogene 18: 3098-3103, 1999.

35. Miyazono K, ten Dijke P and Heldin CH: TGF-beta signaling by Smad proteins. Adv Immunol 75: 115-157, 2000.

36. Rahimi RA and Leof EB: TGF-beta signaling: a tale of two responses. J Cell Biochem 102: 593-608, 2007.

37. Shi Y and Massagué J: Mechanisms of TGF-beta signaling from cell membrane to the nucleus. Cell 113: 685-700, 2003.

38. Derynck R and Zhang YE: Smad-dependent and Smad-independent pathways in TGF-beta family signalling. Nature 425: 577-584, 2003.

39. Lutz M and Knaus P: Integration of the TGF-beta pathway into the cellular signalling network. Cell Signal 14: 977-988, 2002.

40. Massagué J and Chen YG: Controlling TGF-beta signaling. Genes Dev 14: 627-644, 2000.

41. Patil S, Wildey GM, Brown TL, Choy L, Derynck R and Howe PH: Smad7 is induced by CD40 and protects WEHI 231 B-lymphocytes from transforming growth factor-beta -induced growth inhibition and apoptosis. J Biol Chem 275: 38363-38370, 2000.

42. Yamamura Y, Hua X, Bergelson S and Lodish HF: Critical role of Smads and AP-1 complex in transforming growth factor-betadependent apoptosis. J Biol Chem 275: 36295-36302, 2000.

43. Landström M, Heldin NE, Bu S, et al: Smad7 mediates apoptosis induced by transforming growth factor beta in prostatic carcinoma cells. Curr Biol 10: 535-538, 2000.

44. Mazars A, Lallemand F, Prunier C, et al: Evidence for a role of the JNK cascade in Smad7-mediated apoptosis. J Biol Chem 276: 36797-36803, 2001.

45. Hoshino Y, Katsuno Y, Ehata S and Miyazono K: Autocrine TGF-beta protects breast cancer cells from apoptosis through reduction of BH3-only protein, Bim. J Biochem 149: 55-65, 2011.

46. Samanta D, Gonzalez AL, Nagathihalli N, Ye F, Carbone DP and Datta PK: Smoking attenuates transforming growth factor-betamediated tumor suppression function through downregulation of Smad3 in lung cancer. Cancer Prev Res 5: 453-463, 2012.

47. Houde N, Chamoux E, Bisson M and Roux S: Transforming growth factor-beta1 (TGF-beta1) induces human osteoclast apoptosis by up-regulating Bim. J Biol Chem 284: 23397-23404, 2009.

48. Ohgushi M, Kuroki S, Fukamachi H, et al: Transforming growth factor beta-dependent sequential activation of Smad, Bim, and caspase-9 mediates physiological apoptosis in gastric epithelial cells. Mol Cell Biol 25: 10017-10028, 2005.

49. O'Reilly LA, Cullen L, Visvader J, et al: The proapoptotic BH3-only protein bim is expressed in hematopoietic, epithelial, neuronal, and germ cells. Am J Pathol 157: 449-461, 2000.

50. Park WS, Cho YG, Kim CJ, et al: Hypermethylation of the RUNX3 gene in hepatocellular carcinoma. Exp Mol Med 37: 276-281, 2005. 